\title{
Influencia de Tiwanaku en la calidad de vida biológica de la población prehistórica de San Pedro de Atacama ${ }^{1}$
}

Maria Antonietta Costa J., ${ }^{2}$ W Alter Alves Neves ${ }^{3}$ Y Mark Hubbe ${ }^{4}$

\section{RESUMEN}

Se examinan cuatro momentos de la prehistoria de San Pedro de Atacama, con el objeto de calificar el impacto de la influencia Tiwanaku en una población local. Fueron realizados estudios osteobiográficos en 161 individuos de los cementerios Solcor-3, Coyo-3 y Quitor-6, representativos de los períodos pre-Tiwanaku (Fases Sequitor, Quitor), Tiwanaku (Fase Coyo; auge de influencia altiplánica), Tiwanaku Final (final Fase Coyo) y postTiwanaku (Fase Yaye-Solor). Los resultados de tres de los marcadores usados apuntan a que se verifica una mejoría en la calidad de vida biológica de la población local, traducida en mayor estatura y dimorfismo sexual, menor ocurrencia de caries, de desgaste oclusal y de pérdida de dientes durante la vida del individuo, ello posiblemente debido al incremento en el consumo de proteína animal durante el momento de influencia de Tiwanaku. La hipoplasia sistémica del esmalte dental y la hiperostosis indican, a primera vista, un cuadro opuesto al de un impacto positivo en esta población; sin embargo, es posible pensar que la mayor incidencia de estas variables se deba a que, como resultado de una mejor dieta, los individuos eran más resistentes a los ataques de agentes patogénicos. El último indicador se relaciona con el uso del cuerpo, el cual señala que, al margen de cualquier cambio propiciado por la influencia altiplánica, la población local mantuvo sus tradicionales actividades cotidianas a lo largo de todo el tiempo.

Palabras claves: San Pedro de Atacama - Tiwanaku - Solcor3 - Quitor-6 - Coyo-3 - osteobiografía - estatura - dimorfismo sexual - patologías dentarias - dieta - uso del cuerpo.

\section{ABSTRACT}

We examine four prehistorical moments in San Pedro de Atacama with the purpose of qualifying the impact that Tiwanaku had on the local population. To this end, we carried out osteobiographic studies on 161 individuals from the cemeteries of Solcor-3, Coyo-3 and Quitor-6, which are representative of pre-Tiwanaku (Sequitor and Quitor Phases), Tiwanaku (Coyo Phase; with a rise of altiplano influence), Final Tiwanaku (final Coyo Phase)

Proyecto FONDECYT 1010735.

2 Instituto de Investigaciones Arqueológicas y Museo R. P. Gustavo Le Paige s. j. (IIAM), Universidad Católica del Norte. Casilla 17, Correo San Pedro de Atacama, II Región, CHILE. Email: mcosta@ucn.cl. and post-Tiwanaku (Yaye-Solor Phase). The results for three of the indicators that we used, suggest an improvement in the biological quality of life of the local population, expressed in greater height and sexual dimorphism, the lesser occurrence of caries, occlusal abrasion and loss of teeth during the individual's life, all of this possibly owing to the increase in the consumption of animal protein that took place under Tiwanaku influence. Although, on a first impression, hyperostosis and the systemic hypoplasia of tooth enamel would indicate a negative impact on the population, it is conceivable that the greater incidence of these variables results from an improved diet that made individuals more resistant to attacks by pathogenic agents. The last indicator we used relates to body use, which reveals that, notwithstanding the changes that would have been propitiated by altiplano influence, the local population kept to its traditional daily activities throughout.

Key words: San Pedro de Atacama - Tiwanaku - Solcor3 - Quitor-6 - Coyo-3 - osteobiography - height - sexual dimorphism - dental pathologies - diet - body use.

Recibido: marzo 2004. Manuscrito revisado aceptado: julio 2004.

\section{Introducción}

A lo largo de la prehistoria de San Pedro de Atacama, el período durante el cual el Estado Tiwanaku ejerció su influencia en la región (400900 DC) es visto como el auge de la cultura atacameña, debido al desarrollo cultural y económico observado, especialmente a través de las ofrendas mortuorias encontradas en las tumbas (Le Paige 1965; Llagostera y Costa 1984; Núñez 1992). Este fue un período de prosperidad no solamente para los habitantes de San Pedro, sino que también para la región, la que se constituyó

3 Laboratório de Estudos Evolutivos Humanos, Departamento de Biología, Instituto de Biociências, Universidade de São Paulo, C. P. 11461, 05422.970 São Paulo-SP-BRASIL. Email: waneves@ib.usp.br

4 Laboratório de Estudos Evolutivos Humanos, Departamento de Biología, Instituto de Biociências, Universidade de São Paulo, C. P. 11461, 05422.970 São Paulo-SP-BRASIL. Email: mhubbe@ib.usp.br 
en un nodo importante en la red comercial del Estado altiplánico (Oakland 1992; Kolata 1993) (Figura 1).

A través de la iconografía Tiwanaku, Berenguer (1998) mostró que, a diferencia de otras regiones relacionadas con esa entidad, San Pedro de Atacama no era un área de producción primaria y de suministro de materia prima para la capital altiplánica, que representara una posición más privilegiada en la jerarquía Tiwanaku. Es posible que el Desierto de Atacama sirviese solamente de intermediario en la transacción del cobre venido del Noroeste Argentino. Aunque esa relación no es clara, la falta de iconografía Tiwanaku en el N.O.A. (Torres 1998), desde donde el Estado altiplánico sin dudas obtenía su bronce, asociada a la importancia de San Pedro de Atacama en el ámbito de su esfera comercial, favorecen esa hipótesis.

Por muchos años nuestro equipo ha estado trabajando en investigaciones bioantropológicas sobre las poblaciones prehistóricas de San Pedro de Atacama, tomando como hito la influencia de Tiwanaku en los grupos locales. En este trabajo se ha sintetizado la información dispersa en diversas publicaciones (Costa y Neves 1990; Neves et al. 1996 Ms y 1999; Neves y Costa 1998 y 1999; Costa et al. 1998; Hubbe et al. $2001 \mathrm{Ms)}$, teniendo como objetivo intentar calificar el tipo de impacto que la influencia Tiwanaku tuvo sobre la población de San Pedro de Atacama; en los trabajos anteriormente citados se propuso la hipótesis de que esa influencia tuvo un impacto positivo en la calidad de vida biológica de los atacameños.
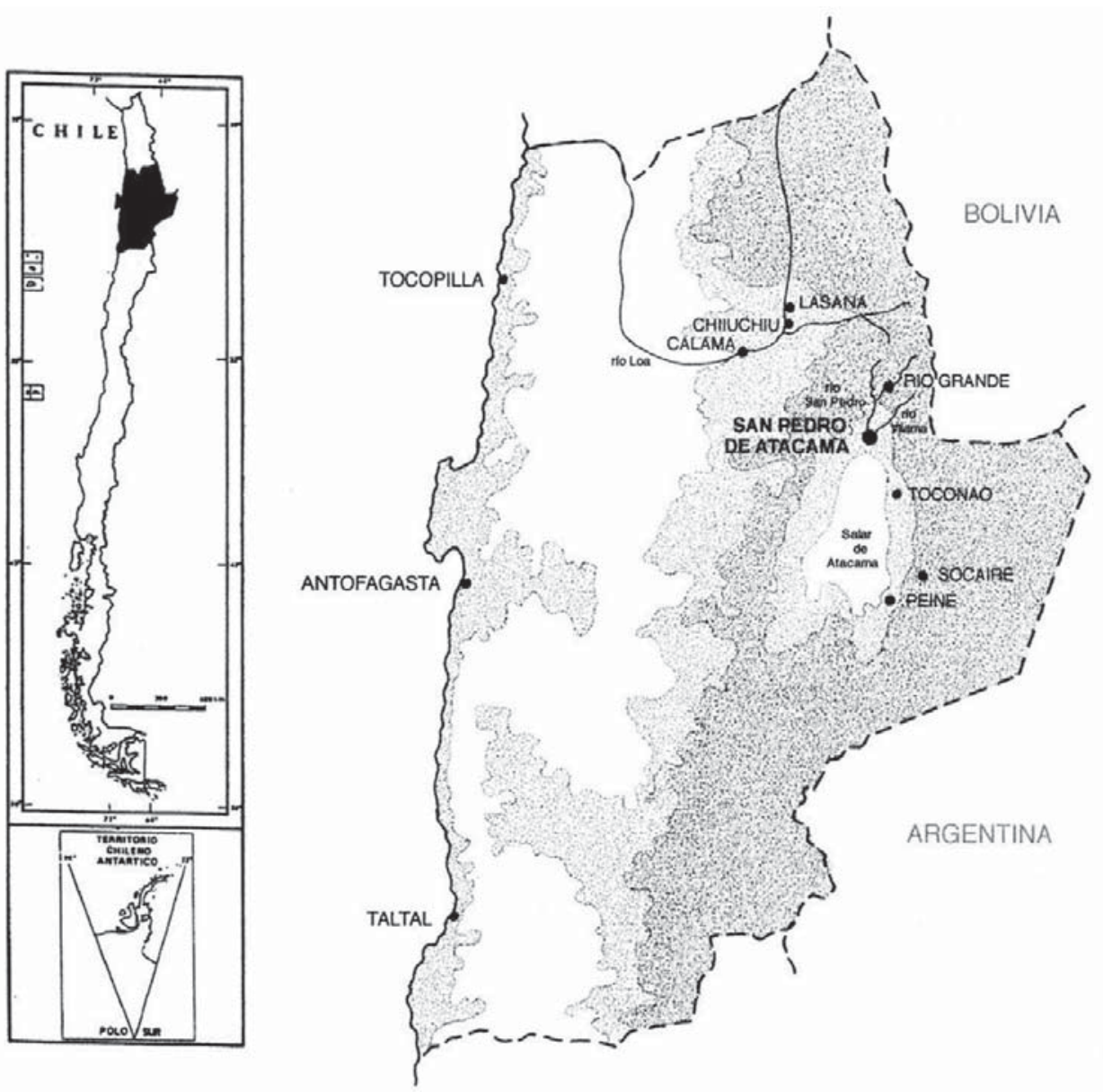

Figura 1. Mapa que muestra la ubicación de San Pedro de Atacama en la II Región de Chile. 


\section{Materiales y métodos}

La muestra considerada la integran 161 individuos adultos, provenientes de tres cementerios (Quitor-6, Coyo-3 y Solcor-3) pertenecientes a cuatro diferentes momentos de la prehistoria san pedrina, incluyendo el período de auge de la influencia de Tiwanaku (Figura 2). Los detalles de la muestra y de sus respectivos fechados pueden ser observados en la Tabla 1. No obstante, el número final de individuos analizados para cada rasgo varió de acuerdo al estado de preservación del esqueleto y de la disponibilidad de las partes anatómicas necesarias. Fueron analizadas las incidencias de siete variables osteológicas consagradas en la literatura, tales como: estatura, a través del largo del fémur; caries; desgaste oclusal; pérdida de dientes in vivo; hipoplasias sistémicas del esmalte dental; hiperostosis porótica y traumas físicos. El objetivo de esos análisis fue caracterizar algunos aspectos socioconductuales y la calidad de vida biológica en cada uno de los períodos, tomando en cuenta aspectos como dieta, nutrición y uso del cuerpo, para evaluar el impacto que la influencia Tiwanaku tuvo sobre la población local.

\section{Largo del fémur}

Actualmente se encuentra consagrada en la literatura especializada la idea de que la estatura de un adulto es un excelente indicador de la calidad nutricional del individuo (Eveleth y Tanner 1976; Tanner 1981 y 1986; Jelliffe 1966; Martorell 1982; Larsen 1987; Frisancho 1990; Steckel 1995; Steckel y Rose 2002), no solamente por el hecho de existir una fuerte correlación entre la estatura y la cantidad y/o la calidad de alimento ingerido (McLaren 1976), sino también porque la variación en la estatura de los adultos refleja, probablemente, condiciones crónicas que inciden en la población (Bogin 1988). En otras palabras, la estatura de los adultos refleja las condiciones ambientales en las cuales el individuo se insertaba durante su fase de crecimiento. En este trabajo, el largo máximo de los fémures fue usado como una aproximación a la estatura del individuo, dada la alta correlación existente entre ambas variables (Maresh 1955; Genovés 1967; Ubelaker 1978; Brothwell 1981; Larsen 1987 y 1999) (Figura 3).

Con el objeto de optimizar el número de fémures disponibles, los momentos Tiwanaku Final y post-

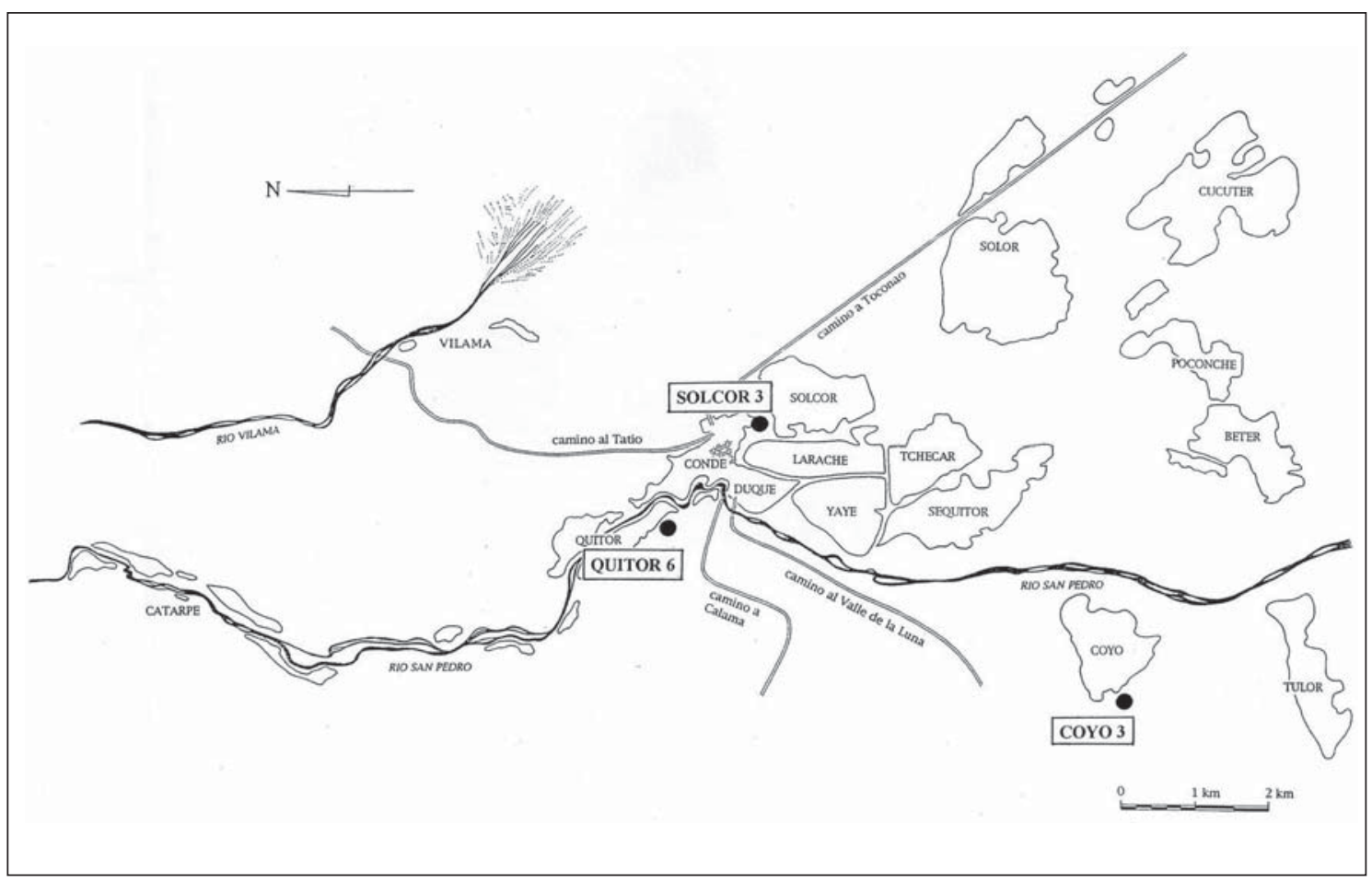

Figura 2. Ubicación de los sitios mencionados en el texto, en la localidad de San Pedro de Atacama. 


\begin{tabular}{|c|c|c|c|c|}
\hline \multirow[b]{2}{*}{ Período } & \multirow[b]{2}{*}{ Sitio } & \multirow[b]{2}{*}{$\mathrm{N}$} & \multicolumn{2}{|c|}{ Fechados absolutos } \\
\hline & & & Cronología & Fuente \\
\hline Pre-Tiwanaku & Solcor-3 & 38 & $\begin{array}{l}250 \pm 150 \mathrm{DC} \\
480 \pm 60 \mathrm{DC}\end{array}$ & $\begin{array}{l}\text { Llagostera et al. } \\
\text { (1988: 64) }\end{array}$ \\
\hline Auge Tiwanaku & Solcor-3 & 41 & $\begin{array}{l}480 \pm 80 \mathrm{DC} \\
570 \pm 60 \mathrm{DC} \\
680 \pm 90 \mathrm{DC} \\
510 \pm 150 \mathrm{DC}^{\mathrm{b}} \\
720 \pm 95 \mathrm{DC}^{\mathrm{b}} \\
850 \pm 110 \mathrm{DC}^{\mathrm{b}} \\
910 \pm 50 \mathrm{DC}^{\mathrm{b}} \\
920 \pm 120 \mathrm{DC}^{\mathrm{b}}\end{array}$ & $\begin{array}{l}\text { Llagostera et al. } \\
(1988: 65) \\
\text { Berenguer et al. } \\
(1988: 344)\end{array}$ \\
\hline Tiwanaku Final & Coyo-3 & 33 & $\begin{array}{l}910 \pm 70 \mathrm{DC} \\
920 \pm 80 \mathrm{DC} \\
960 \pm 50 \mathrm{DC}\end{array}$ & $\begin{array}{l}\text { Costa y Llagostera } \\
\text { (1994: 75) }\end{array}$ \\
\hline Post-Tiwanaku & Quitor-6 & 49 & $\begin{array}{r}920 \pm 70 \mathrm{DC} \\
1060 \pm 180 \mathrm{DC} \\
1240 \pm 70 \mathrm{DC}\end{array}$ & Costa (1988: 105) \\
\hline
\end{tabular}

Tabla 1. Muestra esqueletal analizada y su cronología. A menos que se indique lo contrario, todos los fechados son radiocarbónicos. b: Fechados por termoluminiscencia.

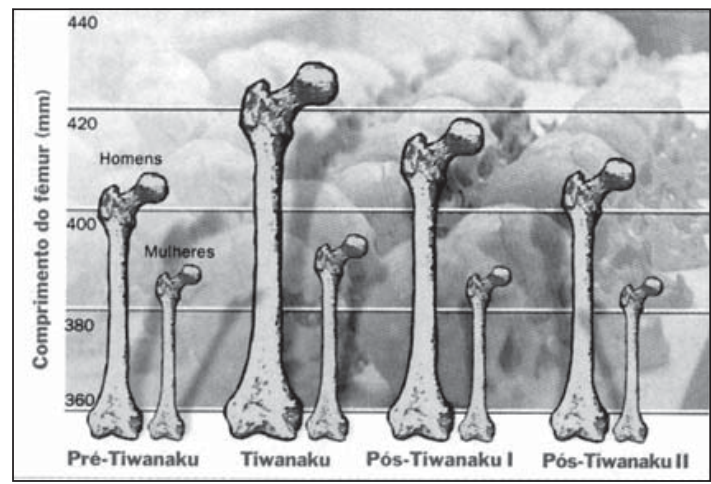

Figura 3. Longitud de fémures masculinos y femeninos a través de los períodos considerados en el análisis.

Tiwanaku fueron reunidos en un único grupo, denominado post-Tiwanaku. Los largos de los fémures de cada período fueron comparados para cada uno de los sexos separadamente, para evaluar el grado de dimorfismo sexual, dado que la estatura femenina parece ser bien menos afectada por estreses nutricionales agudos que la estatura masculina (Tanner 1962; Stini 1969, 1982, 1985). Las diferencias fueron comparadas a través del test $\mathrm{T}$ de Student.

\section{Caries, desgaste oclusal y pérdida de dientes in vivo}

Fueron analizados la incidencia de caries, el desgaste oclusal y la pérdida de dientes in vivo en cada una de las muestras. Las caries, por ser una demineralización del esmalte dentario resultante de la fermentación por acción bacteriana, presentan una alta correlación con la cantidad de carbohidratos ingeridos por un individuo (Hillson 1996; Larsen 1999). Esa asociación ha sido muy utilizada como indicador de dieta en poblaciones prehistóricas (Turner 1979; Hillson 1979 y 1996; Newbrun 1982; Powell 1985; Larsen 1987). La incidencia de caries (según el criterio de Turner 1979) fue calculada para cada uno de los períodos y sus frecuencias fueron comparadas a través de tests de $\chi^{2}$.

De la misma forma, el desgaste oclusal se caracteriza por la erosión natural de la corona dentaria debido al atrito entre los dientes y entre éstos y el alimento ingerido (Molnar 1971 y 1972; Hillson 1996); este rasgo se muestra altamente correlacionado con la abrasividad de la alimentación ingerida (Molnar 1972; B. H. Smith 1984). Puesto que los alimentos más fibrosos (como los vegetales cuando son comparados con la carne) son los más abrasivos, el grado de desgaste oclusal de las muestras prehistóricas es también muy utilizado 
para la reconstitución de la dieta (P. Smith 1972; Kieser et al. 1985; McKee y Molnar 1988; Molnar y Molnar 1985; Kaifu 1999). El grado de desgaste de cada pieza dental fue evaluado según la escala propuesta por Molnar (1972) y los grados medios de desgaste de cada período fueron comparados a través del test de análisis de varianza de Kruskall-Wallis.

La pérdida de dientes in vivo, a pesar de ser una respuesta a procesos inflamatorios del alvéolo (Hillson 1996), fue usada aquí como un indicador de dieta, dada su acentuada relación tanto con carie como con desgaste (Lukacs 1992). Su incidencia fue calculada de acuerdo con el número de alvéolos vacíos que presentaban señales de reabsorción ósea, dividido por el número total de alvéolos observados. Las frecuencias de cada período fueron comparadas entre sí a través de tests de $\chi^{2}$.

\section{Hiperostosis porótica e hipoplasias sistémicas del esmalte}

Tanto la hiperostosis porótica como las hipoplasias lineares del esmalte ${ }^{5}$ fueron analizadas como indicadores de períodos de estrés nutricional (Huss-Ashmore et al. 1982; Klepinger 1984; Larsen 1987). La mayoría de los estudios sobre la hiperostosis porótica asocia esta patología a la anemia resultante de la deficiencia de hierro, sea debido a dietas deficientes en ese mineral (Klepinger 1992), sea debido a respuestas a infecciones (Stuart-Macadam 1989), o aún a la acción de parásitos hematófagos en el tracto digestivo, que disminuyen la cantidad de sangre en el cuerpo (Larsen 1999). Sea cual fuere el principal agente causador de la hiperostosis en las series esqueletales analizadas, esta patología es una respuesta fisiológica del cuerpo a algún tipo de estrés ambiental. Fue analizada la incidencia de hiperostosis solamente en los techos de las órbitas, y las frecuencias de cada período fueron comparadas a través del test exacto de Fischer.

A diferencia de la hiperostosis, las hipoplasias no son asociadas a ninguna etiología específica y, de esta forma, su incidencia ha sido muy empleada como marcador de estrés fisiológico acentuado no

5 Definiciones detalladas de ambas patologías pueden ser encontradas en Larsen (1999). específico (Huss-Ashmore 1982; Klepinger 1984; Goodman et al. 1984; Skinner y Goodman 1992). En este trabajo fueron analizadas solamente las hipoplasias sistémicas en los dientes anteriores de la arcada superior y, en su ausencia, en sus análogos en la arcada inferior. Las hipoplasias sistémicas son caracterizadas como tal cuando ocurren en más de un diente en el mismo período de desarrollo del individuo (Goodman et al. 1984). Para minimizar la influencia del desgaste oclusal en la incidencia de hipoplasias fueron analizados solamente los dientes con por lo menos dos tercios de sus coronas presentes. Las frecuencias de hipoplasias en cada período fueron comparadas a través del test exacto de Fischer.

\section{Traumas físicos}

Se realizó la observación en búsqueda de fracturas cicatrizadas visibles a simple vista, usando el trabajo de Ortner y Putschar (1981) como referencia. Las fracturas ocurridas alrededor del período perimortem no fueron analizadas, debido a la dificultad de su identificación. La incidencia de fracturas fue analizada para todo el esqueleto y fueron construidas tablas de frecuencia para cada período. La mayoría de las fracturas que ocurre en el esqueleto durante la vida de un individuo puede ser resultado de accidentes de la vida diaria, así que son utilizadas como indicadores del uso que el individuo daba a su cuerpo en sus actividades del día a día. Diferente es el caso de las fracturas en el cráneo, las que son consideradas predominantemente como indicadores de violencia interpersonal (Merbs 1989; Walker 1982 y 1990) y fueron consideradas aquí como indicadores de violencia en cada período. Las frecuencias de fracturas fueron comparadas a través del test exacto de Fischer.

\section{Resultados}

La Tabla 2 presenta el largo medio de los fémures de las parcelas femenina y masculina de cada período, así como sus desviaciones estándar, coeficientes de variación y de dispersión. En primer lugar, se observa una acentuada diferencia entre el tamaño de los fémures entre ambas parcelas. Para los tres períodos analizados, esas diferencias son muy significativas $(\mathrm{p}<0.001)$. Entre los períodos se observan además, variaciones en el largo del fémur, tanto en la parcela masculina como en la femenina, siendo que el período de influencia 
Tiwanaku presenta mayores largos que los demás períodos. Entretanto, la única diferencia significativa encontrada fue entre la parcela masculina de los períodos de influencia Tiwanaku y preTiwanaku, cuando los individuos masculinos que vivieron durante el tiempo de influencia altiplánica eran significativamente más altos que los que vivieron antes. La disminución en el largo de los fémures en el período post-Tiwanaku presenta también una baja probabilidad de ser debida al azar $(\mathrm{p}=0.051)$, no significativa $(\mathrm{p}<0.05)$. El hecho de que el coeficiente de variación de cada período no difiera mucho de los demás, sugiere que el aumento de estatura durante Tiwanaku ocurrió en la totalidad de la parcela masculina y no solamente en una élite. Las mujeres, por otro lado, no demuestran diferencias significativas en los largos de sus fémures a través de los períodos analizados, demostrando que la influencia de Tiwanaku no tuvo un impacto tan fuerte en esa parcela de la población.

En la Tabla 3 puede ser observada la incidencia de caries en cada uno de los cuatro períodos analizados, y en las parcelas femenina y masculina de cada uno de ellos. Mirando la incidencia en la población como un todo, se observa una altísima frecuencia de esa patología en la población a lo largo del tiempo. El período menos afectado corresponde al del auge de la influencia Tiwanaku, presentando el $33.6 \%$ de piezas con caries, mientras que el período más afectado es el post-Tiwanaku, con más de la mitad de los dientes cariados. Estos altos índices, especialmente cuando son comparados con los de otras poblaciones horticultoras del mundo (Turner 1979; Larsen 1987), muestran que los atacameños estuvieron ingiriendo siempre alimentos extremadamente cariogénicos.

De todos modos, se observan diferencias significativas entre los períodos estudiados con los períodos correspondientes al auge de influencia y al final de la influencia Tiwanaku significativamente menos afectados que los demás. Se desprende de lo anterior que, dada la relación entre caries y carbohidratos en la generación de caries, a lo largo de la prehistoria san pedrina habría existido una menor contribución de carbohidratos en la dieta local durante los dos períodos bajo influencia Tiwanaku.

\begin{tabular}{|c|c|c|c|c|c|}
\hline Período & $\mathrm{N}$ & Media & $\begin{array}{l}\text { Desviación } \\
\text { Estándar }\end{array}$ & $\begin{array}{l}\text { Coeficiente de } \\
\text { Variación (\%) }\end{array}$ & Rango \\
\hline \multicolumn{6}{|l|}{ Pre-Tiwanaku } \\
\hline Hombres & 14 & 415.28 & 13.76 & 3.31 & $397-437$ \\
\hline Mujeres & 11 & 387.82 & 15.62 & 4.03 & $355-421$ \\
\hline \multicolumn{6}{|l|}{ Tiwanaku } \\
\hline Hombres & 16 & $427.69 *$ & 17.48 & 4.09 & $404-462$ \\
\hline Mujeres & 18 & 393.67 & 17.15 & 4.36 & $361-429$ \\
\hline \multicolumn{6}{|l|}{ Post-Tiwanaku } \\
\hline Hombres & 21 & 413.14 & 24.37 & 5.90 & $371-450$ \\
\hline Mujeres & 36 & 387.92 & 17.67 & 4.55 & $350-430$ \\
\hline
\end{tabular}

Tabla 2. Estadística descriptiva para el largo del fémur en cada período.

* Significativamente diferente $(\mathrm{p}<0.05)$ del período anterior.

\begin{tabular}{|c|c|c|c|c|c|c|c|c|}
\hline & \multicolumn{2}{|c|}{ Pre-Tiwanaku } & \multicolumn{2}{|c|}{ Auge Tiwanaku } & \multicolumn{2}{|c|}{ Tiwanaku Final } & \multicolumn{2}{|c|}{ Post-Tiwanaku } \\
\hline & $\mathrm{N}$ & $\%$ & $\mathrm{~N}$ & $\%$ & $\mathrm{~N}$ & $\%$ & $\mathrm{~N}$ & $\%$ \\
\hline Hombres & $100 / 228$ & 43.9 & $69 / 304$ & $22.7 *$ & $98 / 378$ & 25.9 & $105 / 219$ & $47.9^{*}$ \\
\hline Mujeres & $52 / 106$ & 49.1 & $112 / 235$ & 47.7 & $101 / 241$ & $41.9^{*}$ & $170 / 360$ & $55.6^{*}$ \\
\hline Total & $152 / 334$ & 45.5 & $181 / 539$ & $33.6^{*}$ & $199 / 619$ & 32.1 & $275 / 525$ & $52.4 *$ \\
\hline
\end{tabular}

Tabla 3. Frecuencia de caries en las poblaciones. N: Número de dientes afectados sobre total de dientes disponibles.

* Significativamente diferente $(\mathrm{p}<0.05)$ del período anterior. 
Comparando las incidencias de caries entre las parcelas masculina y femenina, se observa que los hombres son menos afectados en todos los períodos. No obstante, esas diferencias sólo se muestran significativas $(\mathrm{p}<0.001)$ en los dos períodos de menor incidencia de caries. La comparación entre los sexos de cada período, especialmente durante los períodos de influencia Tiwanaku, sugiere que los hombres tenían mayor acceso a la proteína animal y, por lo mismo, menor ingestión de fuentes de carbohidratos. Esta interpretación es muy posible, sobre todo si se considera el rol que tiene el hombre en la obtención de carne como recurso alimentario en sociedades tradicionales (para ejemplos, ver Bates 1991).

La Tabla 4 presenta los resultados referentes al desgaste oclusal en cada período y en las parcelas masculina y femenina de cada uno de ellos. Se verifica la ocurrencia de una disminución en el grado medio de desgaste en el período de auge de influencia Tiwanaku, aumentando en el período Tiwanaku Final para volver a disminuir durante el período post-Tiwanaku. En este análisis no fueron observadas diferencias significativas, sea entre períodos, sea entre parcelas sexuales de cada período, aun cuando durante el auge de influencia Tiwanaku se haya presentado el menor grado medio. Entretanto, un análisis más refinado del desgaste dentario en cada período, no presentado en detalle aquí (Hubbe et al. 2001 Ms), mostró que durante el auge de influencia Tiwanaku se verifica una mayor incidencia de desgaste leve y una menor incidencia de desgaste severo que los demás sitios. Ambas diferencias obtuvieron significancia estadística $(\mathrm{p}<0.05)$.

De esta forma, la población atacameña tuvo durante el período de mayor influencia Tiwanaku un mayor acceso a fuentes de alimentos menos fibrosos (menos abrasivos), como la carne, por ejemplo, o pasaron menos tiempo masticando alimentos fibrosos, atenuados por formas más intensas de preparación preoral. Debido a los resultados obtenidos con las caries, es más probable que el menor desgaste esté relacionado con un mayor acceso a la proteína animal. Por otro lado, el hecho de que el período Tiwanaku Final -que también presenta baja incidencia de caries- exhiba tasas tan acentuadas cuanto a las del período preTiwanku, no nos permite descartar completamente la hipótesis de que la influencia Tiwanaku trajo consigo nuevas formas de preparación preoral de los alimentos fibrosos, haciéndolos menos abrasivos, prácticas que habrían sido abandonadas con la disolución de la influencia altiplánica a lo largo del período que llamamos Tiwanaku Final.

Los resultados obtenidos para la pérdida de dientes in vivo (Tabla 5) corroboran los datos obtenidos para caries y desgaste, en el sentido de que el

\begin{tabular}{|lccccccccc|}
\hline & \multicolumn{2}{c}{ Pre-Tiwanaku } & \multicolumn{2}{c}{ Auge Tiwanaku } & \multicolumn{2}{c}{ Tiwanaku Final } & \multicolumn{3}{c|}{ Post-Tiwanaku } \\
& $(1)$ & $(2)$ & $(1)$ & $(2)$ & $(1)$ & $(2)$ & $(1)$ & $(2)$ \\
\hline \multirow{2}{*}{ Hombres } & $750 / 197$ & 3.8 & $881 / 290$ & 3.0 & $1471 / 327$ & 4.5 & $761 / 191$ & 4.0 \\
Mujeres & $388 / 92$ & 4.2 & $611 / 202$ & 3.0 & $796 / 196$ & 4.1 & $810 / 260$ & 3.1 \\
\hline Total & $\mathbf{1 1 3 8 / 2 8 9}$ & $\mathbf{4 . 0}$ & $\mathbf{1 4 9 2 / 4 9 2}$ & $\mathbf{3 . 0}$ & $\mathbf{1 7 0 1 / 4 1 2}$ & $\mathbf{4 . 1}$ & $\mathbf{1 5 7 1 / 4 5 1}$ & $\mathbf{3 . 6}$ \\
\hline
\end{tabular}

Tabla 4. Grado medio de desgaste en las poblaciones. (1) suma del desgaste por cantidad de dientes analizados; (2) grado medio de desgaste.

\begin{tabular}{|lccccccccc|}
\hline & \multicolumn{2}{c}{ Pre-Tiwanaku } & \multicolumn{2}{c}{ Tiwanaku } & \multicolumn{2}{c}{ Tiwanaku Final } & \multicolumn{2}{c|}{ Post-Tiwanaku } \\
& $\mathrm{N}$ & $\%$ & $\mathrm{~N}$ & $\%$ & $\mathrm{~N}$ & $\%$ & $\mathrm{~N}$ & $\%$ \\
\hline Hombres & $168 / 434$ & 38.7 & $129 / 477$ & $27.0^{*}$ & $124 / 416$ & 29.8 & $103 / 352$ & 29.3 \\
Mujeres & $246 / 385$ & 63.9 & $305 / 618$ & $49.4^{*}$ & $526 / 959$ & $54.8^{*}$ & $231 / 592$ & $39.0^{*}$ \\
\hline Total & $\mathbf{4 1 4 / 8 1 9}$ & $\mathbf{5 0 . 5}$ & $\mathbf{4 3 4 / 1 0 9 5}$ & $\mathbf{3 9 . 6 *}$ & $\mathbf{6 5 0 / 1 3 7 5}$ & $\mathbf{4 7 . 3 *}$ & $\mathbf{3 3 4 / 9 4 4}$ & $\mathbf{3 5 . 4}$ \\
\hline
\end{tabular}

Tabla 5. Frecuencia de pérdidas de piezas dentarias in vivo. N: Número de dientes afectados sobre total de dientes disponibles.

* Significativamente diferente $(\mathrm{p}<0.05)$ del período anterior. 
período de auge de la influencia Tiwanaku muestra una tasa de pérdida dentaria mucho menor que durante el período pre-Tiwanaku, tanto en el total de la población como en las parcelas femenina y masculina. Dada la alta correlación entre este marcador y la incidencia de caries y de desgaste oclusal (Lukacs 1992), los resultados aquí presentados sirven sólo para apoyar los obtenidos con aquellos dos marcadores. Los hombres presentan una tasa de pérdida de dientes significativamente menor que las mujeres $(\mathrm{p}<0.05)$ en todos los períodos, también de acuerdo con los resultados obtenidos para la incidencia de caries y de desgaste.

En la Tabla 6 se encuentran las incidencias de hiperostosis porótica en cada período, para la población total y para las parcelas masculina y femenina. Aunque se oberve una mayor incidencia de esa patología durante el período de auge de la influencia Tiwanaku, esta no llegó a ninguna significancia estadística, lo mismo sucede con las comparaciones entre períodos o entre parcelas sexuales. Es posible que este resultado sea debido al pequeño número de muestra disponible para el análisis.

El resultado obtenido para las hipoplasias lineales sistémicas del esmalte (Tabla 7), a pesar del aumento observado durante el período de influen- cia Tiwanaku, no presenta diferencias significativas entre los períodos, o entre las parcelas masculina y femenina de cada período, a excepción del post-Tiwanaku, más afectado significativamente que el período anterior. De la misma forma que para la hiperostosis, es posible que este resultado haya sido influenciado por el pequeño número amostral disponible.

La Tabla 8 muestra la incidencia de traumas en diferentes regiones anatómicas del esqueleto para cada período. Llama la atención la mayor incidencia de fracturas en algunas regiones anatómicas post-craneanas, tales como las costillas, los cúbitos, los radios y los metatarsos. Aunque diferentes causas pueden resultar en fracturas como las observadas en esas regiones del esqueleto, su frecuencia parece estar más asociada a caídas y a fracturas debidas a los terrenos accidentados (Ortner y Putschar 1981), especialmente porque la mayor parte de las observadas en el antebrazo $(88.2 \%)$ ocurre en la porción distal de los mismos, típicas de cuando el individuo lleva sus manos hacia adelante para amortiguar una caída. La incidencia de fracturas registradas en las costillas puede deberse tanto a caídas como a golpes, siendo difícil establecer su verdadera causa.

Las fracturas craneanas son también frecuentes, indicando, por lo tanto, elevadas tasas de violen-

\begin{tabular}{|lccccccccc|} 
& \multicolumn{2}{c}{ Pre-Tiwanaku } & \multicolumn{2}{c}{ Tiwanaku } & \multicolumn{2}{c|}{ Tiwanaku Final } & \multicolumn{2}{c|}{ Post-Tiwanaku } \\
& $\mathrm{N}$ & $\%$ & $\mathrm{~N}$ & $\%$ & $\mathrm{~N}$ & $\%$ & $\mathrm{~N}$ & $\%$ \\
\hline \multirow{2}{*}{ Hombres } & $1 / 13$ & 7.7 & $3 / 15$ & 20.0 & $2 / 22$ & 9.1 & $2 / 11$ & 18.2 \\
Mujeres & $2 / 12$ & 16.7 & $5 / 20$ & 25.0 & $2 / 21$ & 9.5 & $1 / 19$ & 5.3 \\
\hline Total & $\mathbf{3 / 2 5}$ & $\mathbf{1 2 . 0}$ & $\mathbf{8 / 3 5}$ & $\mathbf{2 2 . 9}$ & $\mathbf{4 / 4 3}$ & $\mathbf{9 . 3}$ & $\mathbf{3 / 3 0}$ & $\mathbf{1 0 . 0}$ \\
\hline
\end{tabular}

Tabla 6. Frecuencia de la hiperostosis porótica en las poblaciones. N: Número de dientes afectados sobre total de dientes disponibles.

\begin{tabular}{|lccccccccc|}
\hline & \multicolumn{2}{c}{ Pre-Tiwanaku } & \multicolumn{2}{c}{ Tiwanaku } & \multicolumn{2}{c|}{ Tiwanaku Final } & \multicolumn{2}{c|}{ Post-Tiwanaku } \\
& $\mathrm{N}$ & $\%$ & $\mathrm{~N}$ & $\%$ & $\mathrm{~N}$ & $\%$ & $\mathrm{~N}$ & $\%$ \\
\hline \multirow{2}{*}{ Hombres } & $1 / 10$ & 10.0 & $5 / 11$ & 45.5 & $4 / 10$ & 40.0 & $6 / 8$ & 75.0 \\
Mujeres & $1 / 4$ & 25.0 & $5 / 9$ & 55.6 & $2 / 7$ & 28.6 & $9 / 12$ & 75.0 \\
\hline Total & $\mathbf{2} / \mathbf{1 4}$ & $\mathbf{1 4 . 3}$ & $\mathbf{1 0 / 2 0}$ & $\mathbf{5 0 . 0}$ & $\mathbf{6 / 1 7}$ & $\mathbf{3 5 . 3}$ & $\mathbf{1 5 / 2 0}$ & $\mathbf{7 5 . 0}$ \\
\hline
\end{tabular}

Tabla 7. Frecuencia de la hipoplasia del esmalte en las poblaciones. N: Número de dientes afectados sobre el total de dientes disponibles. * Significativamente diferente $(\mathrm{p}<0.05)$ del período anterior. 
INFLUENCIA DE TIWANAKU EN LA CALIDAD DE VIDA...

\begin{tabular}{|lrrrrrrrrrrr|r|}
\hline & \multicolumn{3}{c}{ Pre-Tiwanaku } & \multicolumn{3}{c}{ Tiwanaku } & \multicolumn{3}{c}{ Tiwanaku Final } & \multicolumn{3}{c|}{ Post-Tiwanaku } \\
& $\mathrm{N}$ & $\mathrm{Fa}$ & $\%$ & $\mathrm{~N}$ & $\mathrm{Fa}$ & $\%$ & $\mathrm{~N}$ & $\mathrm{Fa}$ & $\%$ & $\mathrm{~N}$ & Fa & $\%$ \\
\hline Cráneo & 33 & 3 & 9.09 & 36 & 6 & 16.67 & 30 & 6 & 20.00 & 45 & 11 & 24.44 \\
Costilla* & 56 & 12 & 21.43 & 75 & 8 & 10.67 & 62 & 6 & 9.68 & 72 & 11 & 15.28 \\
Esternón & 25 & 1 & 4.00 & 34 & 1 & 2.94 & 32 & 0 & 0.00 & 43 & 0 & 0.00 \\
Clavícula & 61 & 3 & 4.92 & 74 & 2 & 2.70 & 60 & 1 & 1.67 & 87 & 3 & 3.45 \\
Húmero & 66 & 1 & 1.52 & 75 & 0 & 0.00 & 64 & 0 & 0.00 & 91 & 0 & 0.00 \\
Radio & 67 & 2 & 2.99 & 72 & 5 & 6.94 & 65 & 7 & 10.77 & 89 & 3 & 3.37 \\
Cúbito & 68 & 5 & 7.35 & 76 & 3 & 3.95 & 65 & 5 & 7.69 & 92 & 4 & 4.35 \\
Metacarpo* & 66 & 3 & 4.55 & 68 & 2 & 2.94 & 66 & 0 & 0.00 & 87 & 2 & 2.30 \\
Pelvis & 56 & 0 & 0.00 & 77 & 0 & 0.00 & 66 & 1 & 1.52 & 81 & 0 & 0.00 \\
Fémur & 68 & 0 & 0.00 & 78 & 0 & 0.00 & 66 & 3 & 4.55 & 93 & 0 & 0.00 \\
Tibia & 66 & 0 & 0.00 & 74 & 2 & 2.70 & 66 & 0 & 0.00 & 85 & 1 & 1.18 \\
Peroné & 67 & 2 & 2.99 & 73 & 1 & 1.37 & 66 & 0 & 0.00 & 88 & 0 & 0.00 \\
Metatarso* & 62 & 4 & 6.45 & 61 & 5 & 8.20 & 64 & 3 & 4.69 & 89 & 1 & $1.12^{* *}$ \\
\hline
\end{tabular}

Tabla 8. Frecuencia de traumas en las distintas regiones anatómicas para cada período. N: hueso completo; Fa: frecuencia de trauma; $\%$ : Porcentaje. * Se refiere al conjunto de huesos como un todo. ** Significativamente diferente $(\mathrm{p}<0.05)$ del período de auge Tiwanaku.

cia interpersonal en la población atacameña, principalmente porque gran parte de ellas fueron consecuencia de golpes claramente asestados contra el cráneo.

La susceptibilidad de traumas por parte de los atacameños se mantuvo constante a lo largo de los períodos, una vez que todas las comparaciones realizadas no alcanzaron significancia estadística, a excepción de la incidencia de traumas en el metatarso durante el período post-Tiwanaku, la que es significativamente menor que la observada durante el período de auge de esa influencia. La razón de esta diferencia es difícil de explicar.

\section{Impacto de la influencia Tiwanaku}

Como ya fue dicho anteriormente, el objetivo de este trabajo es el de entender cómo la llegada de Tiwanaku a San Pedro de Atacama afectó la calidad de vida biológica de la población local. La hipótesis defendida en nuestros trabajos anteriores es la de que, sobre todo durante el auge de la influencia Tiwanaku, los atacameños experimentaron una mejor calidad de vida biológica, en relación a los demás períodos. Como puede ser observado en los resultados y en la Tabla 9, donde se muestra el ranking de la incidencia de los marcadores para cada período, solamente algunos de esos marcadores corroboran plenamente tal hipótesis.

En primer lugar, la estatura de los hombres aumentó significativamente, sugiriendo, por lo tanto, una mejoría en la calidad nutricional de la población. En las mujeres, esa mejoría no es tan evidente, posiblemente porque ellas son menos susceptibles a variaciones nutricionales que los hombres (Tanner 1962; Stini 1969, 1982, 1985). En asociación a estos resultados, se verifica que la incidencia de caries disminuyó durante el mismo período, así como el desgaste y la pérdida de dientes in vivo, tanto entre hombres como entre mujeres, siendo esta caída más acentuada en los individuos masculinos que en los femeninos. De las muchas posibilidades existentes para explicar este cuadro, favorecemos la de un mayor acceso a proteína animal por parte de los atacameños durante el período. La carne, además de ser menos cariogénica y abrasiva, es también una fuente alimenticia rica en proteínas y energía y, además de ello, es el único elemento que puede explicar, en conjunto, las modificaciones de los tres marcadores osteológicos.

Encontramos dos maneras de explicar un mayor acceso a la proteína animal por parte de los atacameños durante el auge de la influencia Tiwanaku. La primera es que, con la mejoría de la economía local, la crianza de camélidos, fuente de proteína animal para los grupos horticultores atacameños, pudo ser intensificada, facilitando así su consumo. La otra es que, con la intensificación de la red comercial circumpuneña durante este período, los rebaños de camélidos utilizados por los atacameños para transportar mercaderías en varias direcciones, pudieron ser relocalizados para el consumo local. Con la ida y venida de las caravanas, es posible que la población local ya no haya necesitado esos animales con la misma fre- 


\begin{tabular}{|c|c|c|c|c|c|c|c|c|}
\hline & \multicolumn{2}{|c|}{ Pre-Tiwanaku } & \multicolumn{2}{|c|}{ Auge Tiwanaku } & \multicolumn{2}{|c|}{ Tiwanaku Final } & \multicolumn{2}{|c|}{ Post-Tiwanaku } \\
\hline & I & Rank $^{\mathrm{a}}$ & I & Rank $^{\mathrm{a}}$ & I & Rank $^{\mathrm{a}}$ & I & Rank $^{\mathrm{a}}$ \\
\hline Largo del fémur - Masc (mm) & 415.28 & 2 & $427.69 *$ & 1 & - & - & 413.14 & 3 \\
\hline Largo del fémur - Fem (mm) & 387.82 & 3 & 393.67 & 1 & - & - & 387.92 & 2 \\
\hline Caries $(\%)$ & 45.5 & 3 & $33.6^{*}$ & 2 & 32.1 & 1 & $52.4 *$ & 4 \\
\hline Desgaste (grado medio) & 4.0 & 3 & 3.0 & 1 & 4.1 & 4 & 3.6 & 2 \\
\hline Pérdida in vivo (\%) & 50.5 & 4 & $39.6^{*}$ & 2 & $47.3^{*}$ & 3 & $35.4^{*}$ & 1 \\
\hline Hiperostosis porótica (\%) & 12.0 & 3 & 22.9 & 4 & 9.3 & 1 & 10.0 & 2 \\
\hline Hipoplasias del esmalte (\%) & 14.3 & 1 & 50.0 & 3 & 35.3 & 2 & $75.0^{*}$ & 4 \\
\hline Fracturas - cráneo (\%) & 9.1 & 1 & 16.7 & 2 & 20.0 & 3 & 24.4 & 4 \\
\hline Fracturas - postcráneo (\%) & 4.5 & 4 & 3.1 & 2 & 3.5 & 3 & 2.5 & 1 \\
\hline
\end{tabular}

Tabla 9. Ranking de incidencia de los marcadores durante la prehistoria atacameña. ${ }^{\text {a }}$ El ranking refleja, en principio, la calidad de vida biológica de la población expresada por cada marcador, o sea, cuanto menor es el rango, mejor es la calidad de vida biológica en ese período (ver Wood y colaboradores 1992 para posibles alternativas a esa interpretación); I: incidencia de cada marcador; * significativamente diferente del período anterior

cuencia anterior para realizar comercio con las regiones vecinas, como debe haber sido el caso antes y después de la influencia Tiwanaku. En este caso, la localidad de San Pedro de Atacama habría desempeñado, entonces, un rol de punto de distribución. Creemos que esta segunda hipótesis es la más verosímil, tomando en cuenta las condiciones limitantes para una intensificación del pastoreo (agua y pasto) que prevalecían en los bordes del Salar de Atacama.

Ya durante los finales de la influencia Tiwanaku, este panorama no resulta tan claro. Aunque la incidencia de caries haya seguido siendo similar a la del período anterior, el desgaste aumentó sensiblemente, así como la pérdida de dientes in vivo. La estatura de la población también disminuyó, aunque de forma no significativa. Lo más probable es que este mosaico refleja el proceso de desmantelamiento de la red comercial y de la influencia local de Tiwanaku. En el período postTiwanaku el acceso de la proteína animal se hizo nuevamente limitado, aumentando la incidencia de caries con una retomada de la dieta preponderantemente vegetal. No obstante, tanto el desgaste oclusal como la pérdida de dientes in vivo se hicieron menos acentuados. Esta contradicción entre los marcadores es difícil de ser explicada con la información que disponemos en este momento sobre los hábitos de vida de los atacameños.

Los demás marcadores (hiperostosis porótica, hipoplasias sistémicas del esmalte y fracturas), por otro lado, no favorecen la idea de que haya ocu- rrido un impacto positivo local en el contacto entre Tiwanaku y la población de San Pedro. No obstante, tampoco muestran un empeoramiento en la calidad de vida de la población local durante ese período.

El hecho de que la incidencia de hiperostosis porótica no haya disminuido durante el período del auge Tiwanaku puede ser perfectamente explicado en el caso de que esa patología esté, de hecho, más relacionada con la presencia de parásitos intestinales que con propiamente una dieta pobre en hierro. Si este fuera el caso, una dieta más rica en proteína animal no puede, por sí sola, alterar la incidencia de hiperostosis; para ello sería necesario que la presencia de parásitos en la población disminuyera al mismo tiempo.

Aunque fuera una posibilidad, otros dos factores deben ser tomados en cuenta en relación con estos marcadores. El primero es el tamaño reducido de la muestra. Como puede ser observado en las tablas 6 y 7, las frecuencias de esos dos marcadores varía mucho de un período a otro, pero el pequeño número de muestra hace ineficiente cualquier test de significancia. El segundo factor es la etiología de los marcadores. La correlación asumida por nosotros al inicio de este artículo de que ambas patologías son ocasionadas por períodos de estrés nutricional, puede no ser completamente verdadera, como ha sido enfatizada por algunos autores más recientes (Larsen 1999). Tampoco podemos excluir la idea expresada por la "paradoja osteológica" (Wood et al. 1992), según la cual los individuos más afectados por esas pato- 
logías son, en realidad, los más resistentes, porque sobrevivieron lo suficiente para que las infecciones pudiesen alcanzar el esqueleto y manifestarse. Por otro lado, los individuos sin marcas de patologías óseas pueden ser aquellos que, a causa de su débil resistencia al ataque de organismos patogénicos, sucumbieron antes de que las patologías pudieran quedar impresas en los huesos. Es posible, por lo tanto, que el pequeño aumento en la incidencia de hiperostosis porótica en el período de auge Tiwanaku, aunque no significativa, refleje un aumento de la resistencia de esa población a eventos de estrés agudo, y no una disminución de la calidad de vida biológica. La paradoja presentada no puede ser resuelta con los datos disponibles, pero el conjunto de los resultados obtenidos con otros marcadores, sugiriendo una mejor calidad de vida biológica durante el período de influencia Tiwanaku, parece apoyar nuestra interpretación.

Los marcadores más informativos de nuestros análisis son aquellos que tienen una relación directa con el tipo de dieta que está siendo ingerida por el individuo. Los marcadores con etiología multifactorial, o sea, aquellos que presentan una gran cantidad de factores que influyen en su incidencia, se muestran menos informativos, cuando no conflictivos entre sí.

El último marcador analizado, la incidencia de fracturas, nos muestra que a pesar de la influencia positiva de Tiwanaku en la calidad de vida biológica local, la población no cambió significativamente sus hábitos de vida, por lo menos en lo que respecta a los riesgos físicos a los que estaba expuesta diariamente. La influencia altiplánica en el Desierto de Atacama no alivianó los riesgos intrínsecos a las actividades físicas cotidianas de la población local. Como se vio anteriormente, la hipótesis que favorecemos es la de que la inserción de San Pedro de Atacama a la red de intercambio de Tiwanaku proporcionó una reubicación de los recursos locales, especialmente los camélidos, del transporte a la alimentación. El hecho de que la población local no haya cambiado sus actividades diarias durante ese período, fortalece la idea de que dicha influencia en el Salar de Atacama fue bastante limitada, no habiendo modificado dramáticamente el género de vida local, lo que es congruente con los estudios arqueológicos (Bittman et al. 1978; Berenguer et al. 1980; Costa 1984).
En lo que se refiere a los índices de violencia interpersonal, los resultados contradicen la idea consagrada entre arqueólogos de que hubo un aumento significativo de tensión después de la caída de Tiwanaku, propuesta presentada originalmente por Munizaga (1974) y reforzada por Núñez (1992). Aun cuando se observa un aumento progresivo en la incidencia de traumas craneanos, tal como lo hacen esos autores, esos aumentos no son estadísticamente significativos.

Después del colapso de Tiwanaku, la población de San Pedro presentó una sensible pérdida de su calidad de vida biológica, como lo atestiguan los marcadores analizados (caries, hipoplasias, largo de fémures). No sólo se registra una disminución importante de acceso a fuentes de proteína animal durante ese período, como también éste es el único que se presenta significativamente más afectado por hipoplasias lineales de esmalte, estando sujeto, por lo tanto, a una mayor cantidad de períodos de estrés. No obstante, es probable que esa caída en la calidad de vida biológica local no se deba exclusivamente al colapso de Tiwanaku, puesto que entre los años 1000-1400 DC toda América del Sur pasó por un período decreciente de pluviosidad anual, culminando en una acentuada sequía entre 1245-1310 DC (Ortloff y Kolata 1991; Berenguer 2000). Tales condiciones climáticas tuvieron una enorme repercusión en la estructura del Estado Tiwanaku, pudiendo haber sido una de las causas de su caída (Berenguer 2000). De ello no escaparon las regiones desérticas de más al sur; Quitor-6, el cementerio que representa el período post-Tiwanaku, cronológicamente inserto justamente en ese período de sequía, se caracteriza por un "empobrecimiento" de los contextos mortuorios contrastante con los cementerios del período anterior. También en el Noroeste Argentino se observa para el mismo momento el colapso de gran parte de los señoríos locales (González 1998) que, en el pasado, promovieron el alto estatus de San Pedro de Atacama como punto clave en el ámbito de la red de intercambio de Tiwanaku (Llagostera 1996).

Aunque no sea la única hipótesis para explicar los resultados obtenidos, el cuadro construido sobre una mayor ingestión de proteína animal nos parece ser el que mejor integra dichos resultados con los datos arqueológicos disponibles para la región. Las conclusiones aquí formuladas se basan en el supuesto de que las cuatro muestras re- 
presentan un mismo grupo biológico y que, por lo tanto, presentan las mismas predisposiciones genéticas a través del tiempo. Pero, si la influencia Tiwanaku fuera, además, acompañada por una difusión démica para la región del Desierto de
Atacama, nuestra premisa pudiera ser incorrecta. Trabajos sobre evolución, utilizando las mismas series por nosotros estudiadas son, por lo tanto, imprescindibles a corto plazo para la confirmación o no de ese supuesto.

\section{REFERENCIAS CITADAS}

BATES, D. J., 1991. Human adaptive strategies. Editorial McGraw-Hill, Nueva York.

BERENGUER, J., 1998, La iconografía del poder en Tiwanaku y su rol en la integración de zonas de frontera. Boletín del Museo Chileno de Arte Precolombino 7: 19-37.

2000, Tiwanaku. Señores del lago sagrado. Museo Chileno de Arte Precolombino, Santiago.

BERENGUER, J., V. CASTRO y O. SILVA, 1980, Reflexiones acerca de la presencia de Tiwanaku en el norte de Chile. Estudios Arqueológicos 5: 81-93.

BERENGUER, J., A. DEZA, A. ROMAN y A. LLAGOSTERA. 1988. La secuencia de Myriam Tarragó para San Pedro de Atacama: Un test por termoluminiscencia. Revista Chilena de Antropología 5: 17-54.

BITTMAN, B., L. NUÑEZ y G. LE PAIGE, 1978. Cultura Atacameña. Colección Culturas Aborígenes. Departamento de Extensión Cultural, Ministerio de Educación, Santiago.

BOGIN, B., 1988. Patterns of human growth. Cambridge University Press, Cambridge.

BROTHWELL, D. R., 1981. Digging up bones. Cornell University Press, Ithaca.

COSTA, M. A. 1984. Reconstitución física y cultural de la población tardía del cementerio de Quitor-6 (San Pedro de Atacama). Estudios Atacameños 9: 99-126.

-1988. Reconstitución física y cultural de la población tardía del cementerio de Quitor-6 (San Pedro de Atacama). Estudios Atacameños 9: 99-127.

COSTA, M. A. y A. LlagosterA. 1994. Coyo-3: Momentos finales del Período Medio en San Pedro de Atacama. Estudios Atacameños 11: 73-108.

COSTA, M. A. y W. A NEVES, 1990. Osteobiografy and late agriculture social organization at San Pedro de Atacama, Chile. American Journal of Physical Anthropology 81: 208-209.

COSTA, M. A., W. A. NEVES, A. M. BARROS y R. BARTOLOMUCCI, 1998. Trauma y estrés en poblaciones prehistóricas de San Pedro de Atacama, norte de Chile. Chungara 30 (1): 65-74.
EVELETH, P. B. y J. M. TANNER., 1976. Worldwide variation in human growth. Cambridge University Press, Cambridge.

FRISANCHO, A. R., 1990. Anthropometric standards for the assessment of growth and nutritional status. University of Michigan Press, Ann Arbor.

GENOVES, S., 1967. Proportionality of the long bones and their relation to stature among Mesoamericans. American Journal of Physical Anthropology 26: 67-78.

GONZALEZ, A. R., 1998. Cultura La Aguada: Arqueología y diseños. Editorial Filmediciones Valero, Buenos Aires.

GOODMAN, A. H., D. L. MARTINS, G. J. ARMELAGOS y G. CLARK, 1984. Indication of stress from bones and teeth. En Paleopathology at the origin of agriculture, $\mathrm{M}$ N. Cohen y G. J. Armelagos (Eds.), pp. 13-49. Academic Press, Orlando.

HILLSON, S. 1979. Diet and dental disease. World Archaeology 11: $147-162$

1996. Dental anthropology. Cambridge University Press, Cambridge.

HUBBE, M., M. A. COSTA., y W. A NEVES, 2001 Ms. Dental markers and standard of living in the prehistoric Atacama Desert. Enviado a International Journal of Osteoarchaeology.

HUSS-ASHMORE, R., A. H. GOODMAN y G. J. ARMELAGOS, 1982. Nutritional inference from paleopathology. En Advances in archaeological method and theory, vol. 5, M. B. Schiffer (Ed.), pp. 395-474 Academic Press, Nueva York.

JELLIFFE, D. B., 1966. The assessment of nutritional status of the community. American Journal of Physical Anthropology 26: 67-78.

KAIFU, Y. 1999. Changes in the pattern of tooth wear from prehistoric to recent period in Japan. American Journal of Physical Anthropology 109: 485-499.

KIESER, J. A., H. T. GROENENVELD y B. PRESTON, 1985. Patterns of dental wear in the Lengua indians of Paraguay. American Journal of Physical Anthropology 66: 21-29.

KLEPINGER, L. L., 1984. Nutritional assessment from bones. Annual Review of Anthropology 109: 485-499. 
-1992. Innovative approaches to the study of past human health and subsistence strategies. En Skeletal biology of past people: Research methods, S. R. Saunders y M. A. Katzenberg (Eds.), pp. 121-130. Wiley-Liss, Nueva York.

KOLATA, A., 1991. The technology and organization of agricultural production in the Tiwanaku State. Latin American Antiquity 2: 99-125.

1993. The Tiwanaku: Portrait of an Andean civilization Blackwell, Cambridge.

LARSEN, C. S., 1987. Bioarchaeological interpretations of subsistence economy and behavior from human skeletal remains. En Advances in archaeological method and theory, vol. 10, M. B. Schiffer (Ed.), pp. 339-445. Academic Press, Nueva York.

1999. Bioarchaeology: Interpretating behavior from the human skeleton. Cambridge University Press, Cambridge.

LE PAIGE, G., 1965. San Pedro de Atacama y sus zonas. Anales de la Universidad del Norte 4: 1-99.

LLAGOSTERA, A., 1996. San Pedro de Atacama: Nodo de complementariedad reticular. Estudios y Debates Regionales Andinos 91: 17-42.

LlAGOSTERA, A. y M. A. COSTA, 1984. Museo Arqueológico R. P. Gustavo Le Paige, San Pedro de Atacama. Serie Patrimonio Cultural Chileno. Departamento de Extensión Cultural, Ministerio de Educación, Santiago.

LlAGOSTERA, A., M. C. TORRES y M. A. COSTA, 1988. El complejo psicotrópico en Solcor-3 (San Pedro de Atacama). Estudios Atacameños 9: 61-98.

LUKACS, J. R., 1992. Dental paleopathology and agricultural intensification in South Asia: New evidence from Bronze Age Harappa. American Journal of Physical Anthropology 87: $133-150$

MARESH, M. M., 1955. Linear growth of long bones of extremities from infancy through adolescence. American Journal of Diseases of Children 89: 725-742.

MARTORELL, R., 1982. Nutrition and health status indicators. World Bank, Washington D. C.

MCKEE, J. K. y S. MOLNAR, 1988. Measurements of tooth wear among Australian Aborigines II: Intrapopulational variation in patterns of dental wear. American Journal of Physical Anthropology 76: 125-136.

MCLAREN, D. (Ed.), 1976. Nutrition in the community. John Willey, Londres.

MERBS, C. F., 1989. Trauma. En Reconstruction of life form skeleton. M. Y. Iscan y K. A. Kennedy (Eds.), pp. 161190. Alan R. Liss, Inc., Nueva York.

MOLNAR, S., 1971. Human tooth wear, tooth function and cultural variability. American Journal of Physical Anthropology 34: 175-189.
_ 1972. Tooth wear and function: A survey of tooth functions among some prehistoric populations. Current Anthropology 13: 511-525.

MOLNAR, S. e I. MOLNAR, 1985. Observations of dental disease among prehistoric populations on Hungary. American Journal of Physical Anthropology 67: 51-64.

MUNIZAGA, J. 1974. Paleopatología chilena (informe preliminar). Antropología, Nueva Epoca 7: 35-39.

NEVES, W. A. y M. A. COSTA, 1998. Adult stature and standard of living in prehistoric Atacama Desert, Northern Chile. Current Anthropology 39 (2): 278-281.

- 1999. Nutrition and quality of life in prehistoric San Pedro de Atacama, Chile. Antropología Física Latinoamericana 2: 23-40.

NEVES, W., A. M. BARROS y M. A COSTA, 1999. Incidence and distribution of postcranial fractures in the prehistoric population of San Pedro de Atacama, Northern Chile. American Journal of Physical Anthropology 109: 253-258.

NEVES, W. A., M. A. COSTA, R. A. SALM y R. G. CUNHA, 1996 Ms. Osteology of social organization in San Pedro de Atacama: Trauma and social tension. Trabajo presentado al IV Congresso da Associação Latino-Americana de Biologia Antropológica, Buenos Aires.

NEWBRUN, E., 1982. Sugar and dental caries: A review of human studies. Science 217: 418-423.

NUÑEZ, L., 1992. Cultura y conflicto en los oasis de San Pedro de Atacama. Editorial Universitaria, Santiago.

OAKLAND, A., 1992. Textiles and ethnicity: Tiwanaku in San Pedro de Atacama, North Chile. Latin American Antiquity 3: 316-340.

ORTLOFF, C. R. y A. KOLATA, 1991. Climate and collapse: Agroecological perspectives on the decline of the Tiwanaku State. Journal of Archaeological Science 20: 195-211.

ORTNER, D. J. y W. G. PUTSCHAR, 1981. Identification of pathological conditions in human skeletal remains. Smithsonian Institution Press, Washington D. C.

POWEL, M. L., 1985. The analysis of dental wear and caries in dietary reconstruction. En The analysis of prehistoric diets, R. I. Gilbert y J. H. Mielke (Eds.), pp. 307-338. Academic Press, Nueva York.

SKINNER, M. y A. H. GOODMAN, 1992. Anthropological uses of developmental defects of enamel. En Skeletal biology of past people: Research methods, S.R. Saunders y M. A. Katzenberg (Eds.), pp. 121-130. Wiley-Liss, Nueva York.

SMITH, P., 1972. Diet and attrition in the Natufians. American Journal of Physical Anthropology 37: 233-238. 
SMITH, B. H., 1984. Patterns of molar wear in huntergatherers and agriculturalists. American Journal of Physical Anthropology 63: 39-56.

STECKEL, R. H., 1995. Stature and the standard of living. Journal of Economic Literature 33: 417-26.

STECKEL, R. H. y J. C. ROSE, 2002. The backbone of History: Health and nutrition in the Western Hemisphere. Cambridge University Press, Cambridge.

STINI, W. A., 1969. Nutritional stress and growth: Sex difference in adaptative response. American Journal of Physical Anthropology 31: 417-26.

1982. Sexual dimorphism and nutrient reserves. En Sexual dimorphism in Homo sapiens, R. L. Hall (Ed.), pp. 391419. Praeger, Nueva York.

-1985. Growth rates and sexual dimorphism in evolutionary perspective. En The analysis of prehistoric diets, R. I. Gilbert y J. H. Mielke (Eds.), pp. 191-226. Academic Press, Orlando.

STUART-MACADAM, P. L., 1989. Nutritional deficiency diseases: A survey of scurvy, rickets and iron-deficiency anemia. En Reconstruction of life from the skeleton, $\mathrm{M}$. Y. Iscan y K. A. Kennedy (Eds.), pp. 201-222. Alan R. Liss, Inc., Nueva York.

TANNER, J. M., 1962. Growth at adolescence. Blackwell, Oxford.
-1981. A history of the study of human growth. Cambridge University Press. Cambridge.

- 1986. Growth as a mirror of the condition of society: Secular trends and class distinction. En Human growth: A multidisciplinary review, A. Demirjian (Ed.), pp. 3-34. Taylor and Francis, Londres.

TORRES, C. M., 1998. Psychoactive substances in the archaeology of Northern Chile and N.W. Argentina: A comparative review of evidence. Chungara 30 (1): 49-63.

TURNER, C. T., 1979. Dental anthropological indications of agriculture among the Jomon People of Central Japan American Journal of Physical Anthropology 51: 619-636.

UBELAKER, D. H., 1978. Human skeletal remains. En Manuals of archaeology, vol. 2, D. H. Ubelaker (Ed.) Taraxacum, Washington D. C.

WALKER, P. L., 1982. Cranial injuries as evidence for the evolution of prehistoric warfare in Southern Carolina. American Journal of Physical Anthropology 54: 287.

- 1990. Cranial injuries as evidence of violence in prehistoric Southern Carolina. American Journal of Physical Anthropology 80: 313-323.

WOOD, J. W., G. R. MILNER, H. C. HARPENDING y K M. WEISS, 1992. The osteological paradox: Problems of inferring prehistoric health from skeletal samples. Current Anthropology 33: 343-370. 